\title{
On the Selection of Sensor Locations for the Fictitious FRF based Fault Detection Method
}

\author{
Kihong Shin ${ }^{1}$ \\ ${ }^{1}$ Department of Mechanical \& Automotive Engineering, Andong National University, Andong, Korea, \\ kshin@anu.ac.kr
}

\begin{abstract}
Recently a machinery fault detection technique based on the fictitious frequency response function (FRF) was developed especially when a measurement sensor cannot be attached to an ideal point due to geometric or environmental restrictions. The fictitious FRF based fault detection method has the great flexibility in regards to choice of sensor locations such that they can be selected almost arbitrarily. However, the method requires two sensors and assumes low correlation between two simultaneously measured signals within the interested frequency range. This assumption of low correlation is vague in practical applications. In this paper, the fault diagnostic performance of the method is investigated in regards to the correlation coefficient between two measured signals. A practical guideline for the selection of the two sensor locations is then proposed.
\end{abstract}

Key words: Fictitious Frequency Response Function, Correlation Coefficient, Sensor Location, Fault Detection.

\section{INTRODUCTION}

Nowadays, detecting an incipient fault symptom is becoming increasingly important in the area of condition-based maintenance $(\mathrm{CBM})$ of rotating machinery. Accordingly, numerous fault detection techniques have been introduced based on the time-series analysis, statistical and artificial intelligence techniques, and time-frequency analysis [1 3]. Most of these diagnostic methods utilize measured vibration signals. In order to successfully apply such methods, the measurement sensors must be attached as close to the fault component of interest as possible. For example, Ogbonnaya et al. emphasized on the importance of knowing where and how to take vibration readings, and concluded that the measurement sensors should be attached to the bearing caps of the gas turbine engines [4]. Another example is that an acceleration sensor is attached directly on to the gearbox when the narrowband demodulation analysis for detecting localized gear faults is performed [5].
The importance of sensor locations is not limited to condition monitoring area, but includes many other practical engineering applications such as designing smart devices [6, 7]. However, it is not always possible to place measurement sensors on ideal points in most practical situations as a result of geometric restriction or temperature limitation. In such cases, the measurement sensors have to placed somewhere away from the component of interest. As a result, the signal-to-noise ratio of the measured signal may become too low to detect any incipient fault symptom, hence most fault detection methods may not be easily applicable.

Recently, the concept of fictitious frequency response function (FRF) has been developed and applied to various engineering problems [8 - 11]. Particularly, in order to overcome the problems mentioned above, the fictitious FRF based fault detection method has been made using two simultaneously measured vibration signals and proved to be very effective in incipient fault detection [9]. However, the assumption made in this method is that the cross-spectrum between two signals measured during healthy operating condition of a machine is very low in the range of the expected fault frequencies. This assumption is somewhat vague and may result to challenges in the practical application to situation if one lacks any prior knowledge on the expected fault frequencies. That is, two measured signals are correlated in some frequencies while they are not correlated in some other frequencies. Therefore, it can be very complicated and time consuming to examine every expected fault frequency in detail in order to check whether the selected sensor locations are valid.

The aim of this paper is to present a practical and easy guideline for selecting two measurement sensor locations for the fictitious FRF based fault detection method on the basis of the correlation coefficient in the time domain. In practice, as cited above, two simultaneously measured signals possess both correlated and uncorrelated components. For instance, the rotating frequency and its harmonics are correlated components regardless of the location of the two measurement sensors, while the noise dominated frequencies tends to be less correlated as the distance between two sensors widens. 
Kihong Shin, International Journal of Emerging Trends in Engineering Research, 7(11), November 2019, 569 - 575

Consequently, the correlation coefficient between two signals cannot be zero regardless of whether the machine is in healthy conditions or not, but is dependent on the locations of measurement sensors. In other words, the correlation coefficient between two signals is very high when two sensors are closely placed to each other even for noise dominated frequencies. Conversely, the correlation coefficient becomes small enough to apply the fictitious FRF based fault detection method successfully when the two sensors are sufficiently placed away from each other.

In this paper, the correlation coefficient for various pairs of sensors is examined using the same experimental setup as in [9], and the fault diagnostic performance is compared based on the sensor pairs. Consequently, it was demonstrated that the correlation coefficient in the time domain could be used as a good practical guideline for determining the sensor locations for the fictitious FRF based fault detection method.

\section{A BRIEF REVIEW OF THE FICTITIOUS FRF BASED FAULT DETECTION METHOD}

This section briefly reviews the principle concept of the fictitious FRF based fault detection method [9]. Consider Figure 1 that shows a single input and two outputs model, which corresponds to two arbitrarily selected sensor locations. Let $X_{1}(f)$ and $X_{2}(f)$ be the frequency representation of measured signals in healthy operating condition. If two measurement sensors are placed sufficiently away from each other, the correlation between two signals is very low (but not perfectly uncorrelated) for noise dominated frequency components. The cross-spectral density function of those frequencies can therefore be written as

$S_{X_{1} X_{2}}(f) \approx 0$

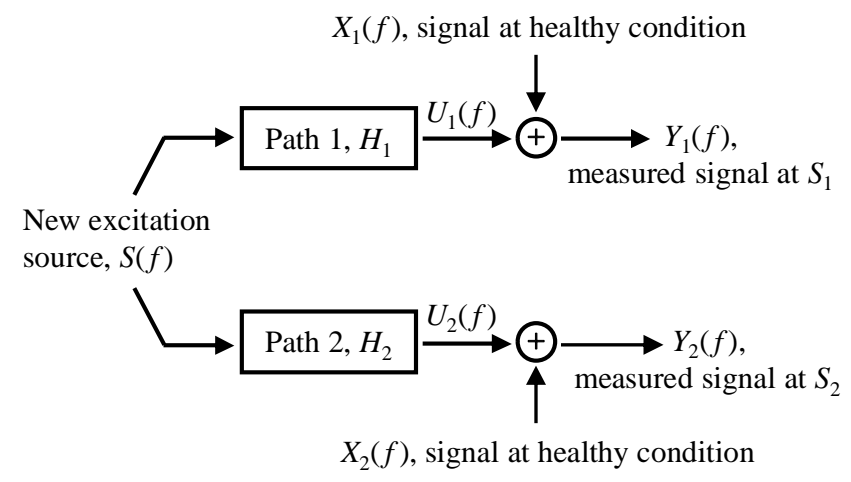

Figure 1: Single input - two outputs model

Later, if a new excitation source, $S(f)$ occurs as a result of a new localized fault within the previously noise dominated frequency region, then the newly measured signals $Y_{1}(f)$ and $Y_{2}(f)$ at the same measurement points contain both uncorrelated noise signals at healthy condition and correlated new fault signals as shown in Figure 1. Therefore, the cross-spectral density function between two signals at this frequency component can be written as

$$
S_{Y_{1} Y_{2}}(f) \approx H_{1}^{*}(f) H_{2}(f) S_{s s}(f)
$$

Although the cross-spectrum expressed in equation (2) contains the fault source power, the fault symptom may not be easily noticeable due to a poor signal-to-noise ratio especially in the case of an incipient fault. The fictitious FRF based fault detection method solves this problem by utilizing a fictitious input-output relationship between two cross-spectral density functions as shown in Figure 2.

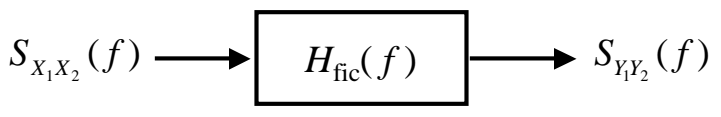

Healthy condition A fictitious FRF A new fault

Figure 2: A fictitious input-output model

The fictitious FRF, $H_{\text {fic }}(f)$, informs the difference between the healthy state and the faulty state of the machine, and can be written as

$H_{\text {fic }}(f)=\frac{S_{Y_{Y_{2}}}(f)}{S_{X_{1} X_{2}}(f)}=\frac{H_{1}^{*}(f) H_{2}(f) S_{s s}(f)}{S_{X_{1} X_{2}}(f)}$

The denominator of equation (3), $S_{X_{1} X_{2}}(f)$, is very small in the healthy condition as described in equations (1). This means that the magnitude of the fictitious FRF may be greatly amplified if there is a new fault source power, $S_{s s}(f)$. This demonstrates that the fictitious FRF based fault detection method can be very useful in monitoring early fault even when the ideal sensor points are unavailable.

However, if the denominator of equation (3), $S_{X_{1} X_{2}}(f)$, is large, i.e., two signals are correlated to some degree at that frequency, the fault symptom may not be detected until the fault source power, $S_{s s}(f)$ becomes strong enough. Thus, the right choice of the sensors location is an important practical aspect.

\section{FAULT DETECTION PERFORMANCE AND CORRELATION COEFFICIENTS FOR VARIOUS SENSOR PAIRS}

As mentioned in the previous section, a pair of sensor locations should be carefully selected for the fictitious FRF based fault detection method in order to detect any fault symptoms as early as possible. Since there is no analytic approach of finding the appropriate sensor locations, in this 
paper, the use of correlation coefficient in the time domain is investigated by examining the fault diagnostic performance based on the correlation coefficients calculated for various cases of sensor pairs.

The same experimental setup is used as in [9], however, a couple of more sensor locations are considered as shown in the following section. It is validated that the correlation coefficient could easily be utilized as practical guideline of judging whether the selected location of the two sensors is appropriate or not.

\subsection{Description of the Experimental Setup}

Consider an experimental setup as shown in Fig. 3 where a rotor is driven by a servo AC motor at $30 \mathrm{~Hz}(1800 \mathrm{rpm})$ and is supported by two ball bearings. Four measurement points are considered: one accelerometer $\left(S_{1}\right)$ is attached on the far-end bearing housing while three accelerometers $\left(S_{2} \sim S_{4}\right)$ are attached on the base frame as shown in the figure 3 .

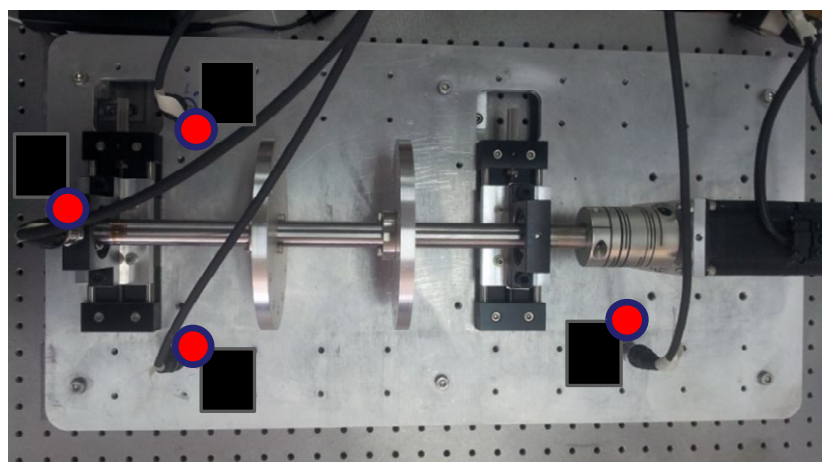

Figure 3: Experimental setup and measurement points

Two fault cases at the far-end bearing are considered: one on the outer race and the other on the inner race of the bearing. The bearing fault characteristic frequency of each case is given by [12]

BPFO (Ball-pass frequency, outer race)

$$
=\frac{n f_{r}}{2}\left(1-\frac{d}{D} \cos \phi\right)
$$

BPFI (Ball-pass frequency, inner race)

$$
=\frac{n f_{r}}{2}\left(1+\frac{d}{D} \cos \phi\right)
$$

where $f_{r}$ is the rotating frequency of the rotor, $n$ is the number of balls, $d$ is the ball diameter, $D$ is the pitch diameter, and $\phi$ is the angle of the load from the radial plane. The parameters in this experiment are $f_{r}=30 \mathrm{~Hz}, n=8, d=8 \mathrm{~mm}, D=34.5 \mathrm{~mm}$, and $\phi=0^{\circ}$, and the expected fault characteristic frequencies are $\mathrm{BPFO}=93 \mathrm{~Hz}$ and $\mathrm{BPFI}=147 \mathrm{~Hz}$, respectively.

\subsection{Fault Detection Performance and Correlation Coefficients for Various Sensor Pairs}

Four accelerometer signals were measured simultaneously for each of the three conditions of the far-end bearing: healthy, outer race fault, and inner race fault. A total of six pairs of sensors are considered in the construction of cross-spectral density functions that corresponds to the sensor pairs given by: ' $S_{1} \& S_{2}$ ', ' $S_{1} \& S_{3}$ ', ' $S_{1} \& S_{4}$ ', ' $S_{2} \& S_{3}$ ', ' $S_{2} \& S_{4}$ ', and ' $S_{3} \&$ $S_{4}$.

The cross-spectral density function for the healthy condition is utilized as an input while that of the faulty condition is used as an output when the fictitious FRF is obtained as shown in Figure 2.

The fault detection performance is quantitatively examined for each pair of sensors. First, the magnitude spectrums of the fictitious FRFs in the case of the outer race bearing fault are shown as in Figure 4. The outer race fault characteristic frequency $(93 \mathrm{~Hz})$ is observed for all cases of sensor pairs. However, sensor pairs of ' $S_{1} \& S_{4}$ ', ' $S_{2} \& S_{4}$ ' and ' $S_{3} \& S_{4}$ ' exhibited the fault frequency more distinctively compared to the other three sensor pairs which are relatively close to each other.

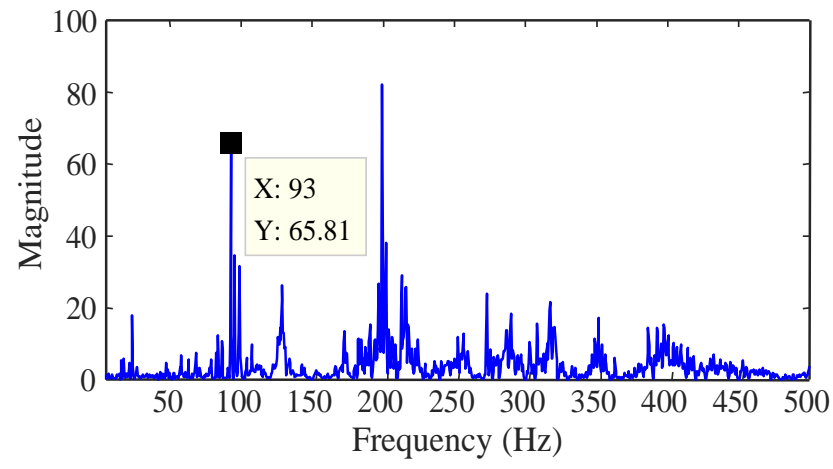

(a) Sensor pair: $S_{1} \& S_{2}$

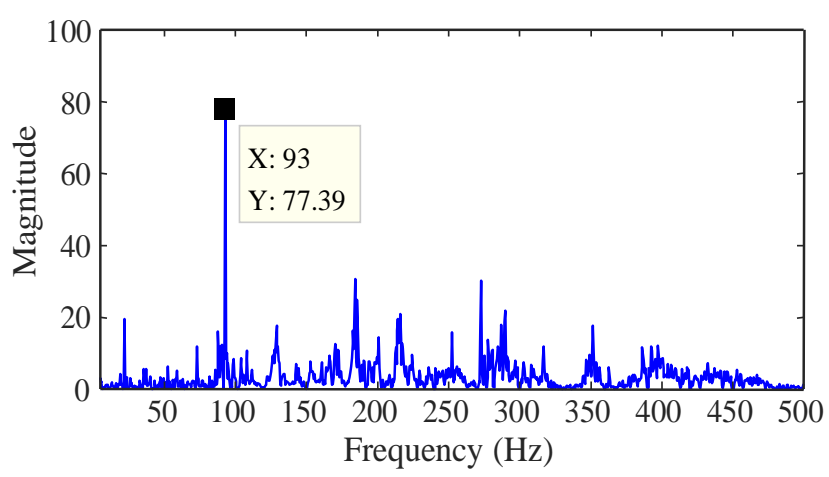

(b) Sensor pair: $S_{1} \& S_{3}$ 


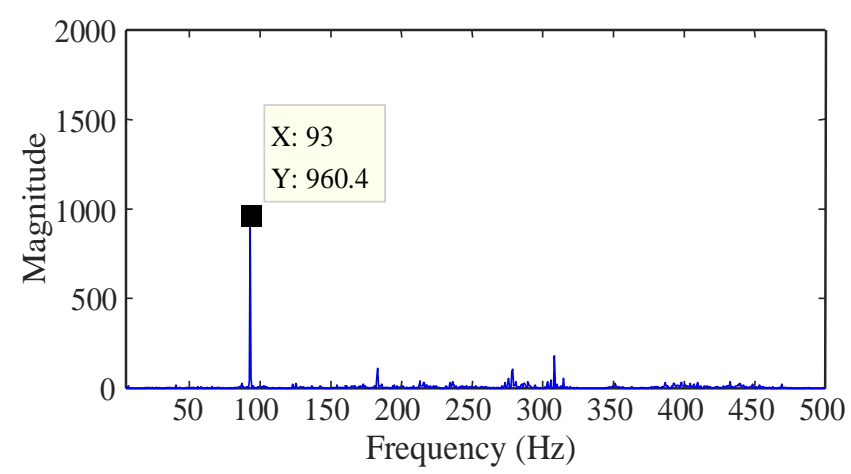

(c) Sensor pair: $S_{1} \& S_{4}$

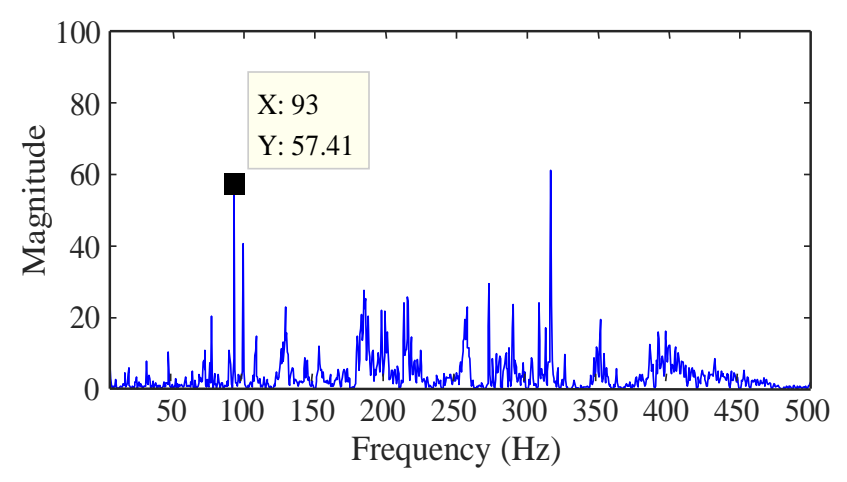

(d) Sensor pair: $S_{2} \& S_{3}$

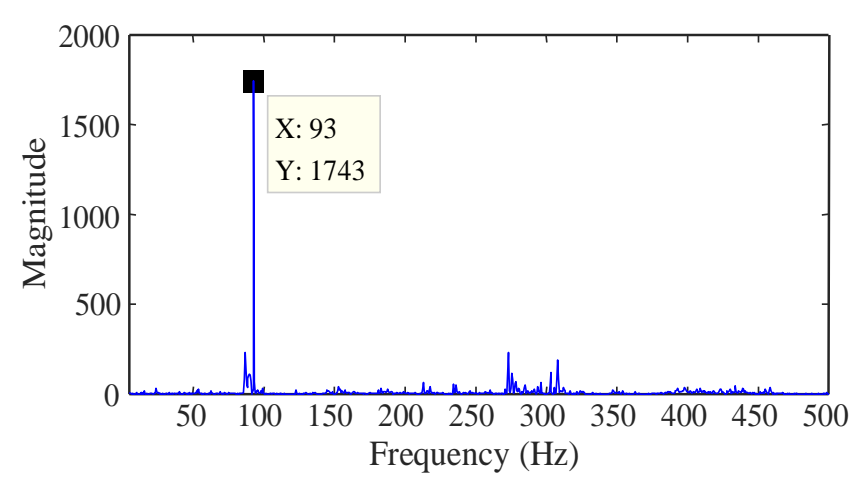

(e) Sensor pair: $S_{2} \& S_{4}$

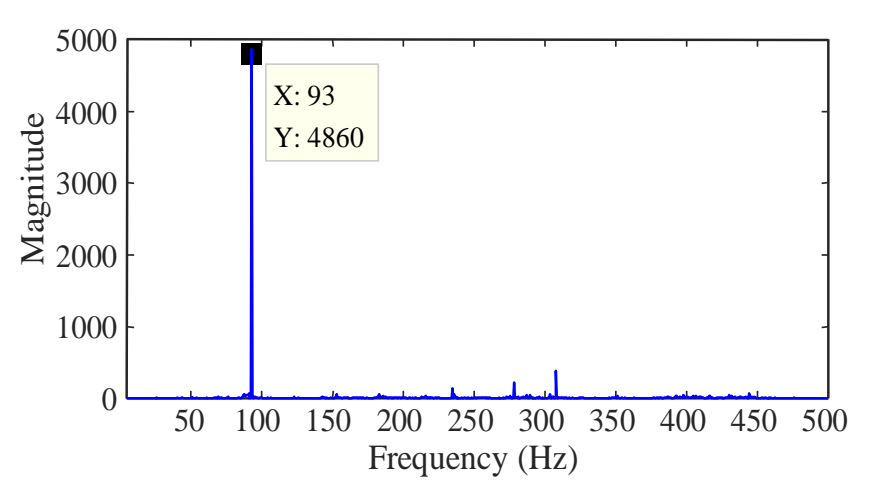

(f) Sensor pair: $S_{3} \& S_{4}$

Figure 4: Magnitude spectrum of the Fictitious FRF in the case of BPFO
Similar results are obtained in the case of inner race bearing fault where the fault characteristic frequency was found to be $147 \mathrm{~Hz}$ as shown in Figure 5. The same sensor pairs as in the case of the outer race bearing fault $\left(S_{1} \& S_{4}, S_{2} \& S_{4}\right.$ and $S_{3} \&$ $S_{4}$ ) reveals the fault frequency better compared to the other sensor pairs. These results indicate that a larger distance between two sensors implies a smaller correlation to the fault characteristic frequency in healthy bearing condition. Specifically, the denominator of equation (3) is small, thus, the magnitude of the fictitious FRF at this fault frequency is greatly amplified for the sensor pairs of ' $S_{1} \& S_{4}$ ', ' $S_{2} \& S_{4}$ ' and ' $S_{3} \& S_{4}$ '. Notably, sensor pair with the largest distance $\left(S_{3} \& S_{4}\right)$ showed the greatest magnitude as shown in Figure 4(f) and Figure 5(f).

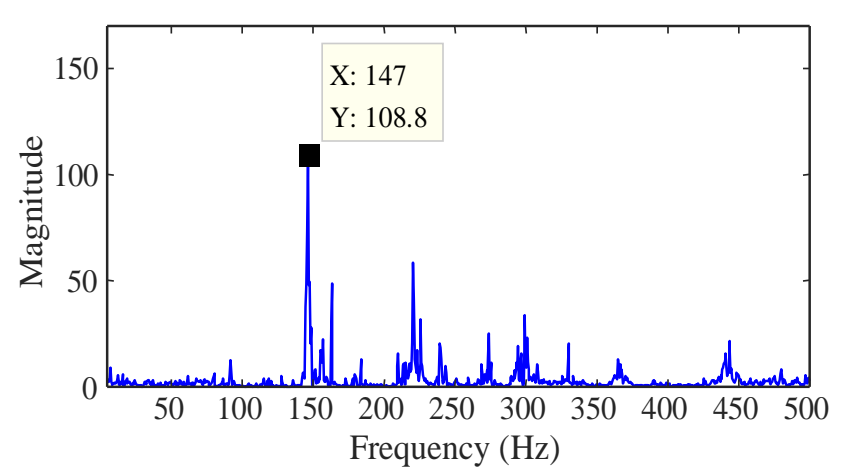

(a) Sensor pair: $S_{1} \& S_{2}$

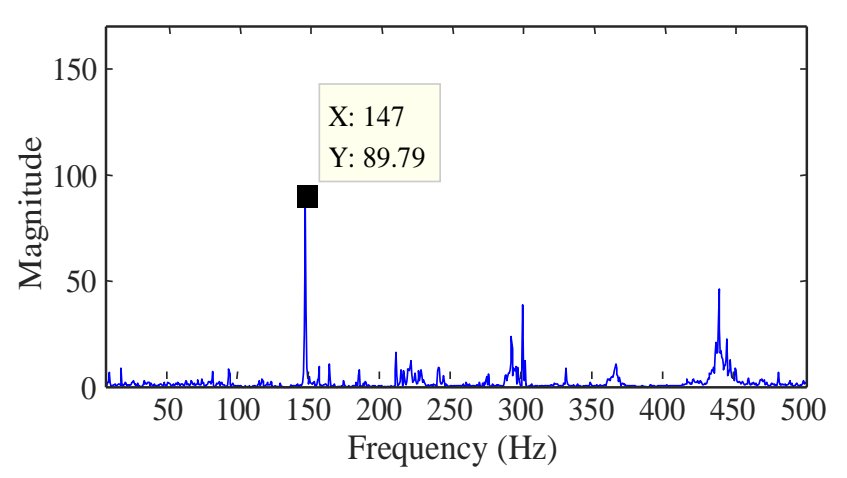

(b) Sensor pair: $S_{1} \& S_{3}$

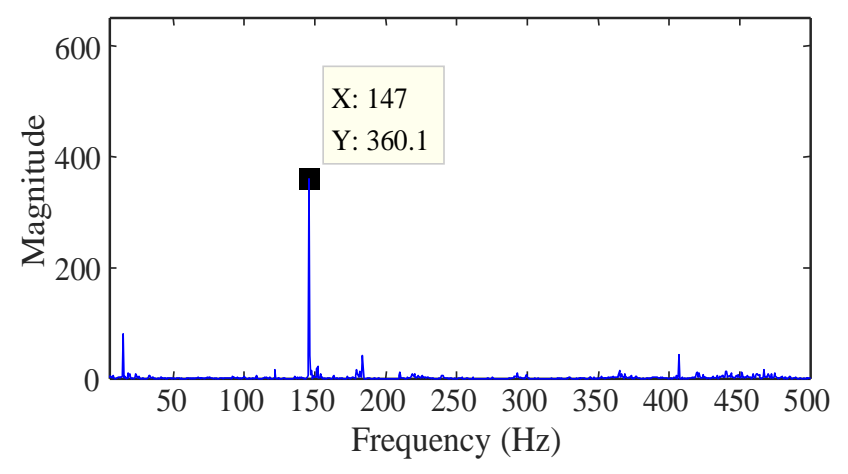

(c) Sensor pair: $S_{1} \& S_{4}$ 
Kihong Shin, International Journal of Emerging Trends in Engineering Research, 7(11), November 2019, 569 - 575

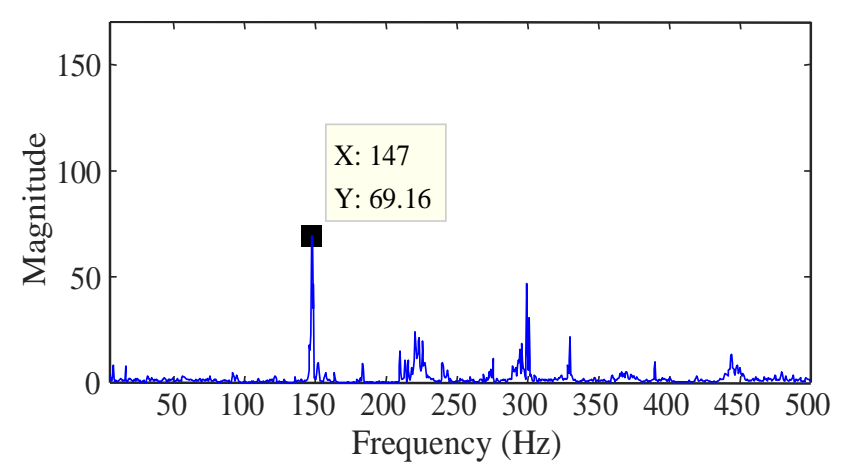

(d) Sensor pair: $S_{2} \& S_{3}$

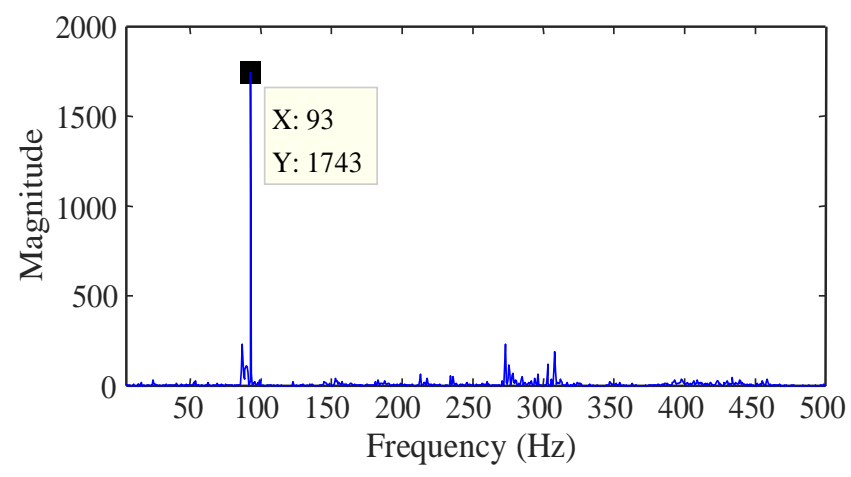

(e) Sensor pair: $S_{2} \& S_{4}$

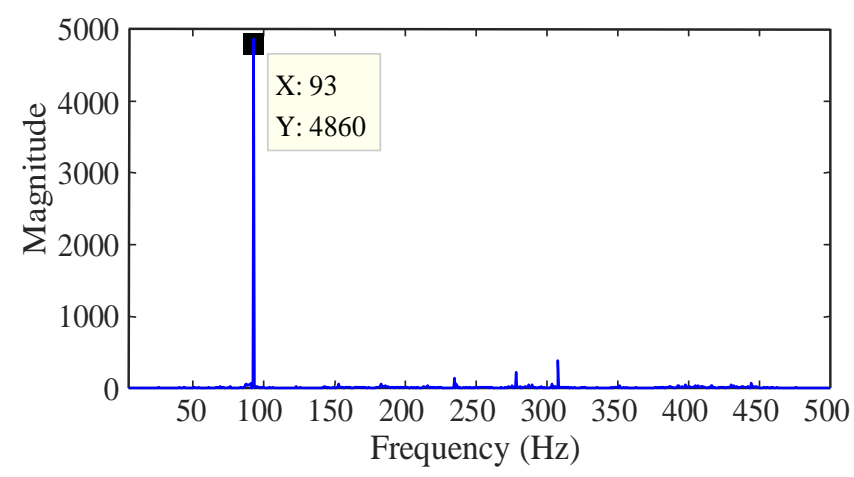

(f) Sensor pair: $S_{3} \& S_{4}$

Figure 5: Magnitude spectrum of the Fictitious FRF in the case of BPFI

From the results shown above, it could be emphasized that the distance between two measurement sensors should be wide enough for better detection of a fault symptom. However, it is not easy to decide whether the selected sensors are located sufficiently far away from each other. Since there is no analytic solution for this, the correlation coefficient calculated in the time domain is investigated whether it could be used as a crucial reference in selection of sensor locations. The correlation coefficient is a measure of the linear relationship between two signals, and is defined as [13].

$$
\rho_{x_{1} x_{2}}=\frac{\operatorname{Cov}\left(x_{1}, x_{2}\right)}{\sigma_{x_{1}} \sigma_{x_{2}}}
$$

where $\operatorname{Cov}\left(x_{1}, x_{2}\right)$ is the covariance between signals $x_{1}(t)$ and $x_{2}(t)$, while $\sigma_{x_{1}}$ and $\sigma_{x_{2}}$ are the standard deviation of the signals. The correlation coefficients between signals corresponding to each sensor pairs for various bearing conditions are calculated and listed in Table 1.

For all bearing conditions, sensor pairs of ' $S_{1} \& S_{4}$ ', ' $S_{2} \& S_{4}$ ' and ' $S_{3} \& S_{4}$ ' showed significantly low values compared to the other sensor pairs, as highlighted in bold font in the table above. These sensor pairs are exactly similar to those which have shown better fault detection performance in Figure 4 and 5 .

Table 1: Sensor pairs and corresponding correlation coefficients for various bearing conditions

\begin{tabular}{|c|c|c|c|c|}
\hline $\begin{array}{c}\text { Sensor } \\
\text { pair }\end{array}$ & $\begin{array}{c}\text { Healthy } \\
\text { condition }\end{array}$ & $\begin{array}{c}\text { Outer-rac } \\
\text { e defect }\end{array}$ & $\begin{array}{c}\text { Inner-rac } \\
\text { e defect }\end{array}$ & Average \\
\hline$S_{1} \& S_{2}$ & 0.754 & 0.712 & 0.730 & 0.730 \\
\hline$S_{1} \& S_{3}$ & 0.736 & 0.683 & 0.781 & 0.733 \\
\hline$S_{\mathbf{1}} \& \boldsymbol{S}_{\mathbf{4}}$ & $\mathbf{0 . 1 9 3}$ & $\mathbf{0 . 1 7 7}$ & $\mathbf{0 . 1 9 4}$ & $\mathbf{0 . 1 8 8}$ \\
\hline$S_{2} \& S_{3}$ & 0.747 & 0.671 & 0.770 & 0.729 \\
\hline $\boldsymbol{S}_{\mathbf{2}} \& \boldsymbol{S}_{\mathbf{4}}$ & $\mathbf{0 . 2 0 4}$ & $\mathbf{0 . 1 4 3}$ & $\mathbf{0 . 2 1 5}$ & $\mathbf{0 . 1 8 7}$ \\
\hline $\boldsymbol{S}_{\mathbf{3}} \& \boldsymbol{S}_{\mathbf{4}}$ & $\mathbf{0 . 1 7 8}$ & $\mathbf{0 . 1 9 2}$ & $\mathbf{0 . 2 6 8}$ & $\mathbf{0 . 2 1 3}$ \\
\hline
\end{tabular}

The differences in correlation coefficients is more clearly visible in Figure 6(a), and the correlation coefficients averaged over three bearing conditions are shown in Figure 6(b). In order to compare the fault detection performance clearly, the magnitude of fault frequency component for each sensor pair is shown in Figure 6(c) for the outer race fault case and in Figure 6(d) for the inner race fault case, respectively.

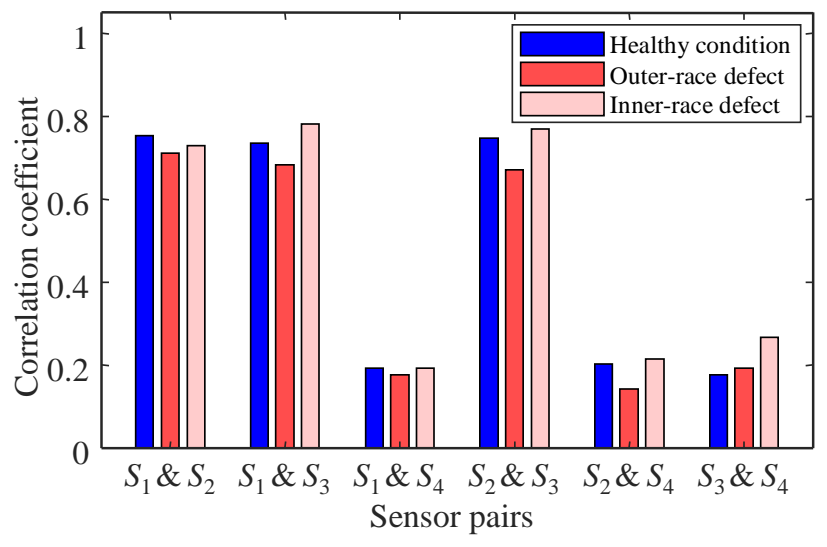

(a) Correlation coefficients 


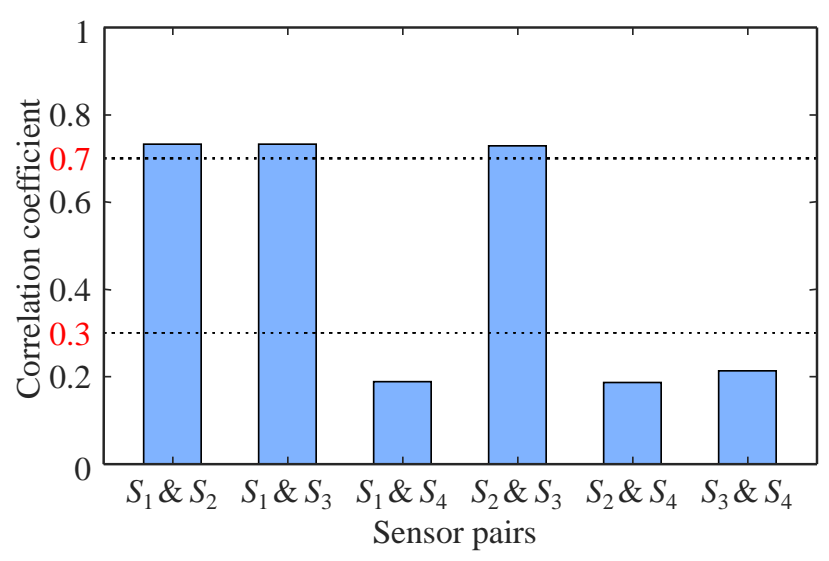

(b) Average value of correlation coefficients

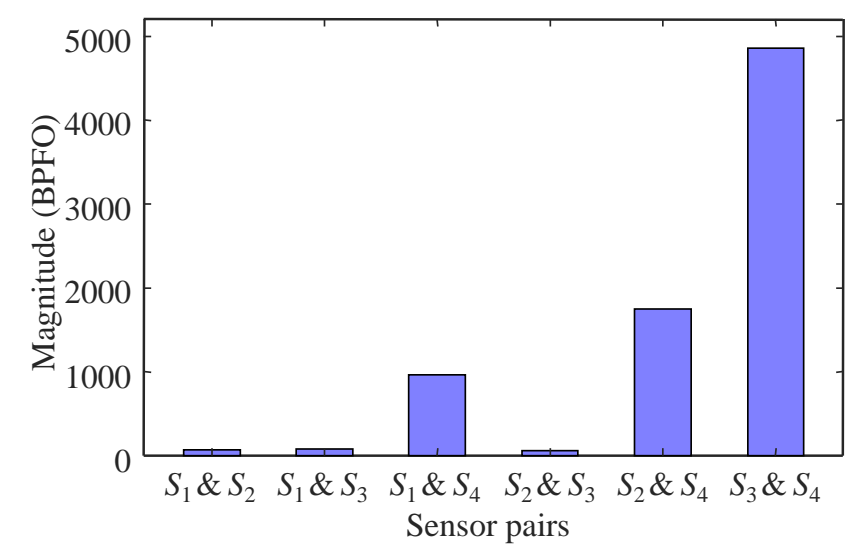

(c) Magnitude of the BPFO component

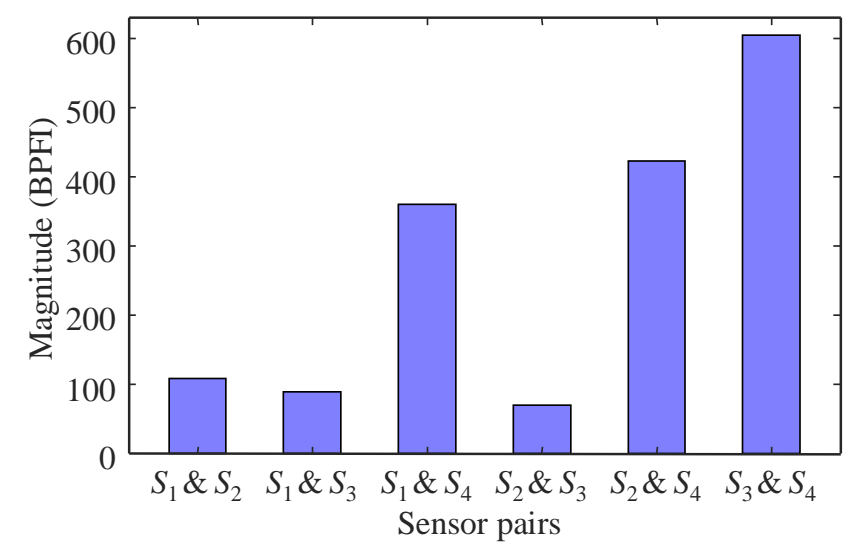

(d) Magnitude of the BPFI component

Figure 5: Magnitude spectrum of the Fictitious FRF in the case of BPFI

Figure 6 indicates that the fault diagnostic performance is greatly related to the correlation coefficient between the two measured signals, such that the sensor pairs having a sufficiently low correlation coefficient guarantee a good fault detection ability. In this specific experiment, the cases which the correlation coefficient was less than 0.3 showed outstanding fault diagnostic performance. Conversely, those with a correlation coefficient of about 0.7 or above showed poorer performance. In this case, although the fault characteristic frequency can be observed in the fictitious FRF, it may not be suitable for detecting an incipient fault symptom.

From the correlation analysis, it may be concluded that a simple calculation of correlation coefficient in the time domain can be utilized as a good criterion to determine whether a selected pair of sensors are located appropriately. Although the experimental results shown in this paper cannot be generalized for all machines, a correlation coefficient lower than 0.3 may be proposed as a useful guideline for selecting sensor locations.

\section{CONCLUSION}

In this paper, the importance of sensor locations is emphasized when applying the fictitious FRF based fault detection method by quantitatively comparing the fault diagnostic performance with respect to the locations of two measurement sensors. Experimental results demonstrate that the distance between the sensors must be large enough such that the correlation coefficient between two measured signals is sufficiently low in order to detect an early fault symptom

For the experiments shown in this paper, correlation coefficient below 0.3 resulted in good diagnostic performance while in cases of correlation coefficient above 0.7 exhibited poorer performance. Even though the relationship between the correlation coefficient and the sensor locations may not be generalized analytically, the result of correlation analysis verifies that the correlation coefficient could be utilized as a crucial practical guideline to judge whether the pair of measurement sensors are located appropriately, and that the values of correlation coefficients shown in this paper may be utilized as a good reference for other applications.

\section{ACKNOWLEDGEMENT}

This work was supported by a Research Grant of Andong National University.

\section{REFERENCES}

1. S. D. Fassois, and J. S. Sakerllariou. Time-series methods for fault detection and identification in vibrating structures, Philosophical Transactions of the Royal Society A, Vol. 365, pp. 411-48, 2007. https://doi.org/10.1098/rsta.2006.1929

2. A. K. S. Jardine, D. Lin, and D. Banjevic. A review on machinery diagnostics and prognostics implementing condition-based maintenance, Mechanical Systems and Signal Processing, Vol. 20, pp. 1483-1510, 2006. 
Kihong Shin, International Journal of Emerging Trends in Engineering Research, 7(11), November 2019, 569 - 575

https://doi.org/10.1016/j.ymssp.2005.09.012

3. Z. Feng, M. Liang, and F. Chu. Recent advances in time-frequency analysis methods for machinery fault diagnosis: A review with application examples, Mechanical Systems and Signal Processing, Vol. 38, pp. 165-205, 2013.

https://doi.org/10.1016/j.ymssp.2013.01.017

4. E. A. Ogbonnaya, E. M. Adigio, H. U. Ugwu, and M. C. Anumiri. Advanced Gas Turbine Rotor Shaft Fault Diagnosis Using Artificial Neural Network, International Journal of Engineering and Technology Innovation, Vol. 3, pp. 58-69, 2013.

5. Y. Guo, Q. N. Liu, X. Wu, and J. Na. Gear Fault Diagnosis Based on Narrowband Demodulation with Frequency Shift and Spectrum Edit, International Journal of Engineering and Technology Innovation, Vol. 6, pp. 243-254, 2016.

6. E. B. Panganiban. Microcontroller-based Wearable Blood Pressure Monitoring Device with GPS and SMS Feature through Mobile App, International Journal of Emerging Trends in Engineering Research, Vol. 7, No. 6, pp. 32-35, 2019. https://doi.org/10.30534/ijeter/2019/02762019

7. W. L. Kim, and S. B. Lim. Smart Chair Cover for Posture Correction, International Journal of Emerging Trends in Engineering Research, Vol. 7, No. 8, pp. 191-196, 2019.

https://doi.org/10.30534/ijeter/2019/14782019

8. K. Shin, H. Yang, S. K. Lee, and Y. S. Lee. Group delay based location template matching method for the identification of the impact location on a plate, Journal of Sound and Vibration, Vol. 332, pp. 2111-2117, 2013. https://doi.org/10.1016/j.jsv.2012.12.007

9. K. Shin, and S. H. Lee. Machinery Fault Diagnosis Using Two-Channel Analysis Method Based on Fictitious System Frequency Response Function, Shock and Vibration, Vol. 2015, Article ID 561238, 2015. https://doi.org/10.1155/2015/561238

10. K. Shin. An alternative approach to measure similarity between two deterministic transient signals, Journal of Sound and Vibration, Vol. 371, pp. 434-4457, 2016.

https://doi.org/10.1016/j.jsv.2016.02.037

11. K. Shin. Development of a degradation index for machinery condition monitoring using the fictitious frequency response function and its application to a centrifugal compressor, International Journal of Condition Monitoring and Diagnostic Engineering Management, Vol. 21, pp. 19-23, 2018.

12. R. B. Randall, and J. Antoni. Rolling element bearing diagnostics - A tutorial, Mechanical Systems and Signal Processing, Vol. 25, pp. 485-520, 2011. https://doi.org/10.1016/j.ymssp.2010.07.017
13. K. Shin and J. K. Hammond. Fundamentals of signal processing for sound and vibration engineers, 1st ed. Chichester, U.K.: Wiley, 2008, ch. 7, pp. 206-211. 